\title{
Solution to optimize the NC programs for milling machining applications
}

\author{
Adrian But $^{1, *}$ and Vlad Botnari ${ }^{2}$ \\ ${ }^{1}$ Politehnica University of Timişoara, Department of Material Engineering and Manufacturing, Mihai \\ Viteazu 1, Timisoara, Romania \\ ${ }^{2}$ Technical University of Moldova, Department of Machine Building Technology, Studentilor 9/8, \\ Kishinev, Republic of Moldova
}

\begin{abstract}
The paper present solutions to optimize the NC program. Using the original NC program, keep the technology with the same cutting tools, the results give us less time of manufacturing with more protection to collisions. The use of new softer with real capabilities solution to manage and optimize the $\mathrm{CNC}$ technologies.
\end{abstract}

\section{Introduction}

When we create an NC program, we must take in consideration more options, more variables and technological aspects to be able to respect the technical drawing of the part, to respect the accuracy of the final product. But in the same time we must think regarding how to increase more profit, we must find the way to do safest and fastest machining and to apply the high technology [1]. This mean to optimize.

In the paper, we try to give an answer regarding to present one solution to resolve these technical problems and to succeed and to have a good and useful NC program on milling centers.

Before NC machining, first we must checklist for the NC program, to do not have machine collision, tool breakage because this is capital facts and can cost a lot of money. One broke NC spindle can cost between few thousands Euro to several tens of thousands of Euro. It maybe be different situation from engineer, so there are variety of human error will occur. Some other important aspects are to establish a correct feed adapted at the particular situations what we have in the manufacturing different parts with different geometry, to calculate the correct RPM, and to manage the convenient air cut and over/ less cutting.

\section{State of the art}

A good solution to give a positive answer at all this requests is to use the advantage of NC BRAIN software (Fig 1). Using NC Brain we can reduces more than $10 \%$ of machining time and $90 \%$ of tool breakage, and helps to increase unmanned machining without worrying about any accident or collision, which will increase run rate after all.

\footnotetext{
*Corresponding author: adi.but@gmail.com
} 


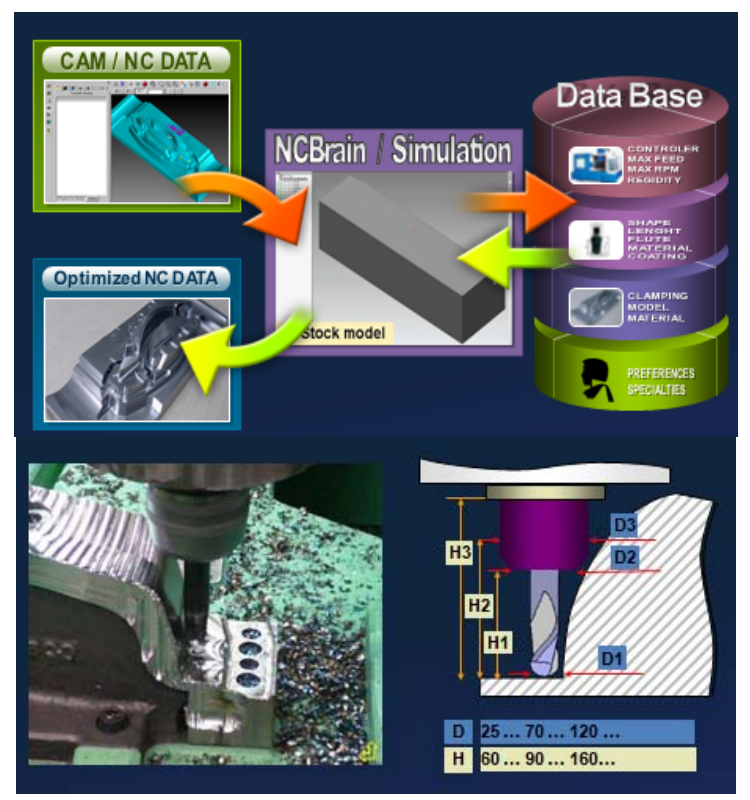

Fig. 1. The philosophy of the NC BRAIN software, and the data base who take in consideration the cutting tools.

$\mathrm{NC}$ data that created by CAM will be regenerated into optimized NC data by simulation and verification. The optimized $\mathrm{NC}$ data makes the safest and fastest machining without any accident.

As we know, not only showing collision between holder and stock in red color and leaving log, but also showing collision between machine and stock, which can easily be corrected as you want. To prevent one small accident will save a lot damage in time and expense after all.
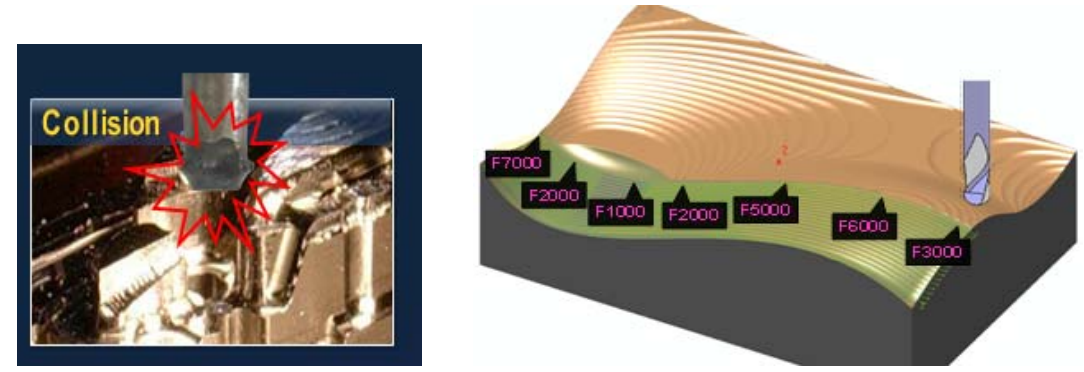

Fig. 2. The prevent collision and the adapted the cutting tools parameters (the feed speed) are the big two advantages of NC BRAIN.

Safe feed rate fit for load may cause excessive machining time. NC Brain controls feed rate on load accordingly and save machining time.

Besides, it will minimize the tool breakage and abrasion. The function mentioned above has been applied to 3 axis and is being applied to simultaneous 5 axis as well which it is the first development in the world. Tool breakage is easily happening on excessive load.

$\mathrm{NC}$ Brain has a function, called 'automatic tool path addition on overloaded area', which prevents tool breakage and deterioration. [3] In particular, this function helps safe machining without any accident during unmanned operation. 


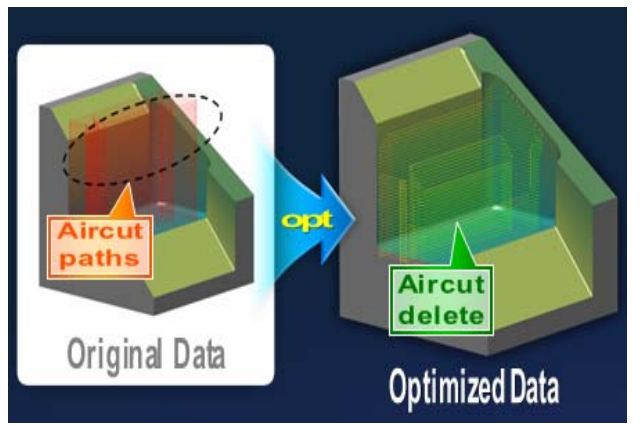

Fig. 3. NC BRAIN function 'automatic tool path addition on overloaded area.

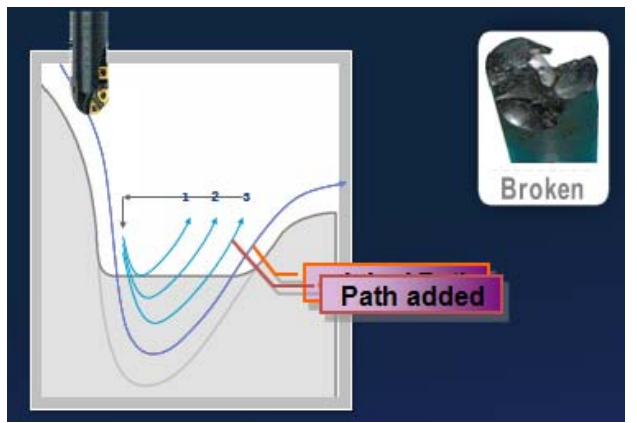

Fig. 4. Data base creation for the cutting tools and the material stock.

In this case will face the waste time of machining on air cut area during operation. Considering this area, will be necessary to create more detailed and stressful CAM. NC BRAIN will save machining time by deleting this air cut area.

Over \& less cutting caused by NC data and wrong tool info will create big damage to the end. This function checks the over $\&$ less cutting by comparing $\mathrm{CAD}$ and simulation model. You can prevent poor faulty in advance with size base and naked eye.

NC BRAIN accuracy on is one hundred-thousandth, and is applicable to the extremely sensitive filed.

Lastly, it is D/B (DATA -BASE) creation. This mean that we will transfer to the software the real information's about the cutting tools, machine tools. This step is very important because with this correct data information's we will have a real feedback from the technological system and a real image about the process. Normally, the most important cutting condition it depends on the engineer. NC BRAIN decides cutting condition by optimization of cutting D/B so we can make fast, safe, and standard machining. NC BRAIN will take in consideration the cutting tools, machines and stock to create $\mathrm{D} / \mathrm{B}$. Consistently, if we will upgrade $\mathrm{D} / \mathrm{B}$, we can have more machining efficiency.

\section{Experimental protocol}

All this advantages we will present on real machining application where we will underline the advantages of NC BRAIN implementation in our work shop. In Figure 5 was presented the CAM solution to manufacturing the part.

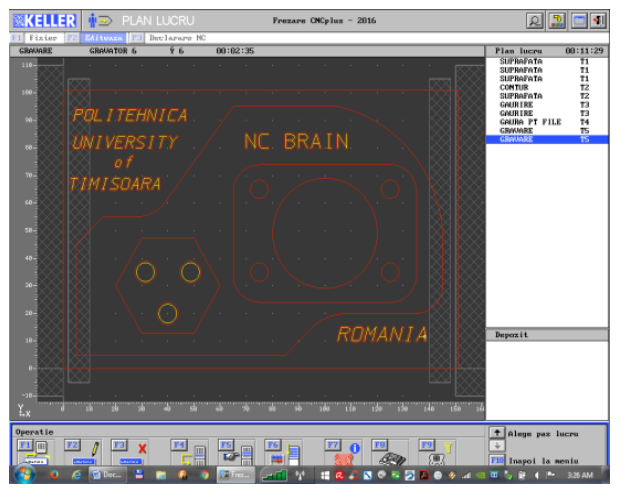

Fig. 5. The CAM application for the part what was manufacturing.

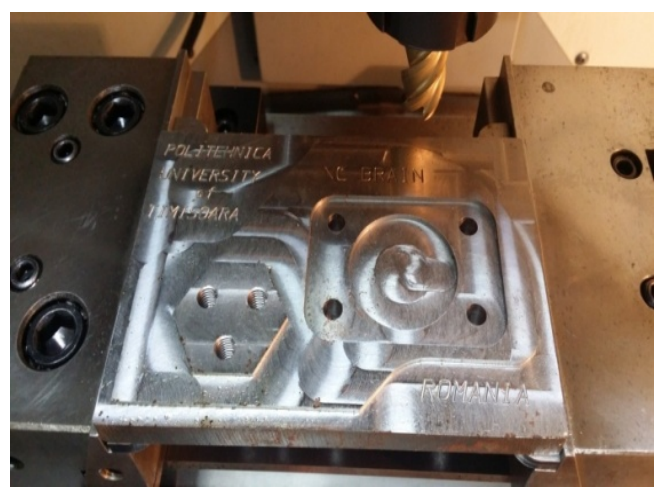

Fig. 6. The real part what was manufacturing. 
We used a raw material: C45, dimensions: 150x100x20 mm. The cutting tools who was used:

1. End mill Ø 18mm, 4 teeth, for manufacturing the inlands and the rectangular and round pockets;

2. End mill $\varnothing 12 \mathrm{~mm}, 4$ teeth, to make the contour in the rectangular and round pockets;

3. Drill $\varnothing 6.8 \mathrm{~mm}$, to manufacturing the drill;

4. Tap M8- for the threaded holes;

5. Engraving tool $\varnothing 6 \mathrm{~mm}$ to engrave the words on the part.

Without using the NC BRAIN we will see the original tool path with green color (Figure 8) and the time manufacturing was 11 minutes and 32 seconds (Figure 7.)

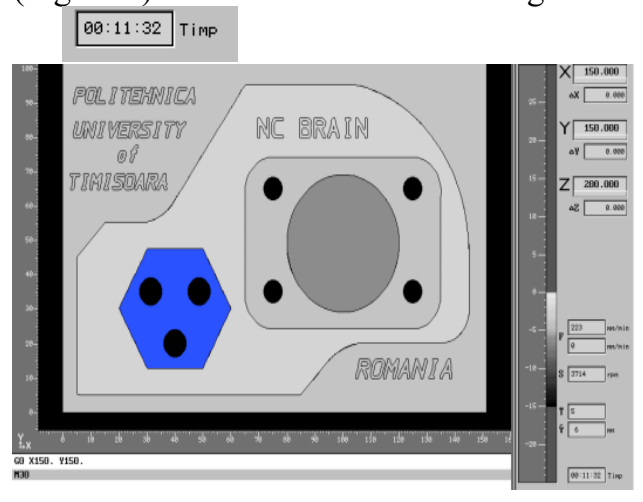

Fig. 7. The final simulation with the manufacturing time: 11 minutes and 32 seconds.

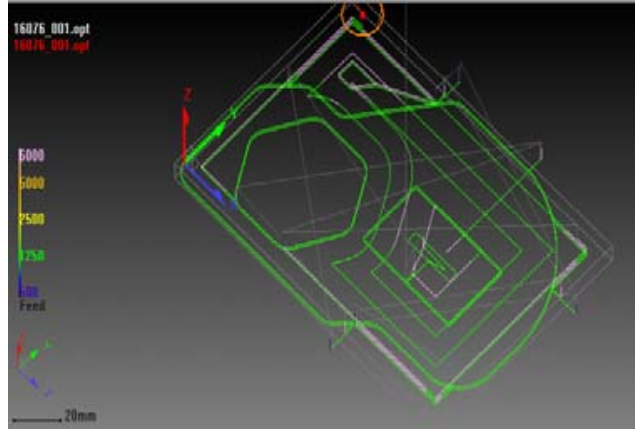

Fig. 8. The green color indicate the original trajectories.

Using the NC BRAIN software we will see different color of the trajectory (yellow, blue, violet) what inform us that was change the feed take in consideration the optimization of the part.

But after the optimization we will see different values of the feed, for example in (Figure 9) we will see in the line 507:

N507 G1 X-3.500 Y41.923 Z-0.599 F6000 S1800

that the values of the feed grow to $6000 \mathrm{~mm} / \mathrm{min}$.

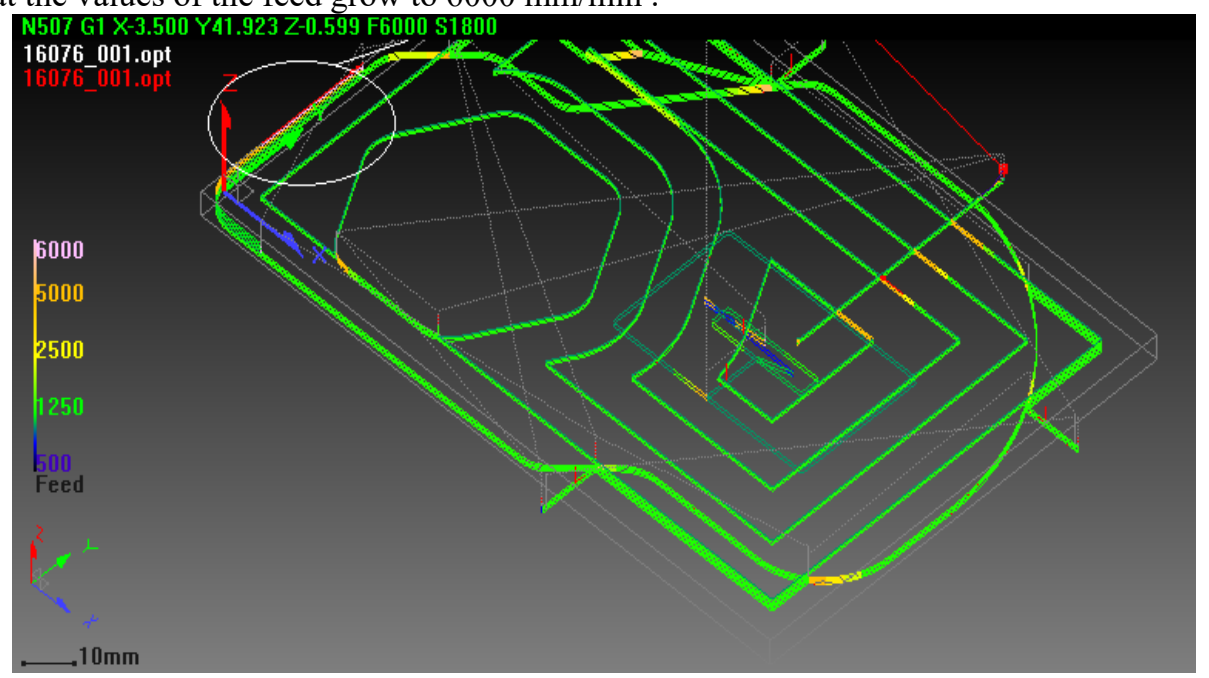

Fig. 9. The line N507 G1 X-3.500 Y41.923 Z-0.599 F6000 S1800 
And in the line N128 the value for the feed is change at $1400 \mathrm{~mm} / \mathrm{min}$. (Figure 10) because here we have one manufacturing process where we must respect the cutting conditions.

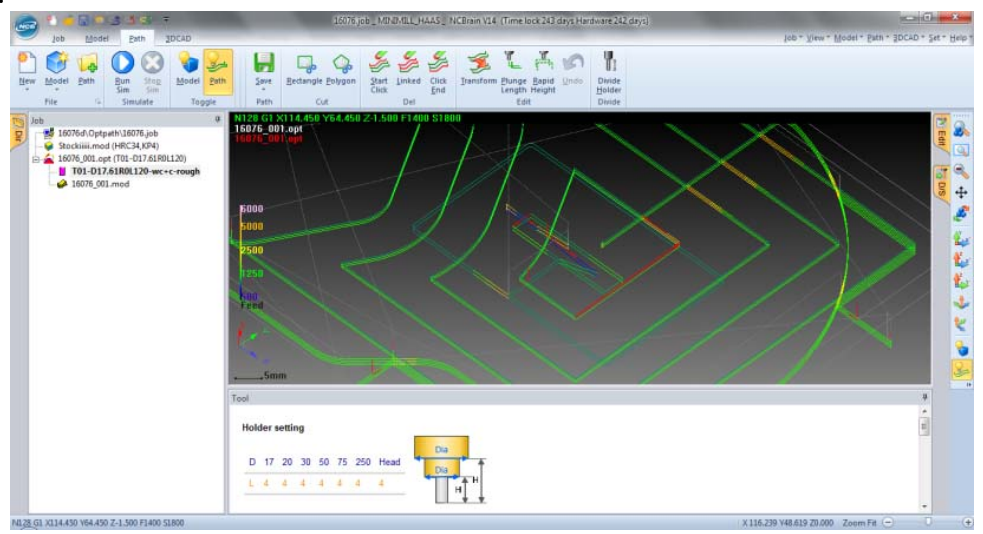

Fig. 10. The line N128 G1 X114.450 Y64.450 Z-1.500 F1400 S1800.

But in the line N797 G1 X83.500 Y49.000 Z -2.444 F670 S1800 the value of the feed was change at $670 \mathrm{~mm} / \mathrm{min}$. (Figure 11).

Here the cutting tools go in the material using the ramp milling strategy and the cutting condition was change.

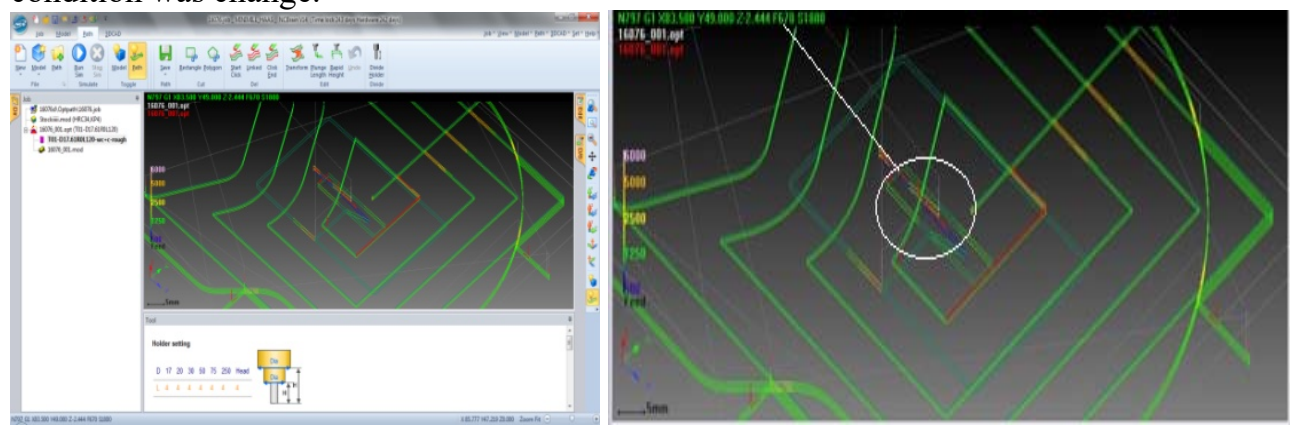

Fig. 11. N797 G1 X83.500 Y49.000 Z -2.444 F670 S1800.

The NC BRAIN can make the difference where the cutting tool is in the milling process and in the situation where we can grow up the feed values where we made the positioning. We can see on Figure 12 that the feed value grow up at $\mathrm{F} 5000 \mathrm{~mm} / \mathrm{min}$.

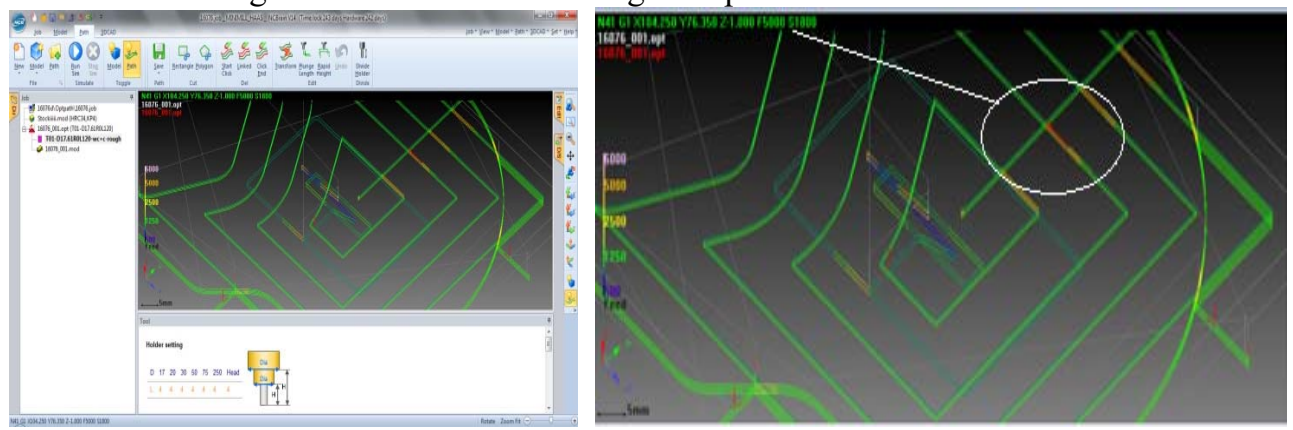

Fig. 12. N41 G1 X10.250 Y76.350 Z-1.000 F5000 S 1800. 


\section{Conclusions}

The test what was made in our workshop for one simple part, using the NC BRAIN software, we obtain 9 minutes and 20 seconds of manufacturing time in comparison with 11 minutes and $32 \mathrm{sec}$. without the implementation of this software. (Figure13)

So, the benefit was with $19 \%$ less time of manufacturing using the same machine tools, the same cutting tools.

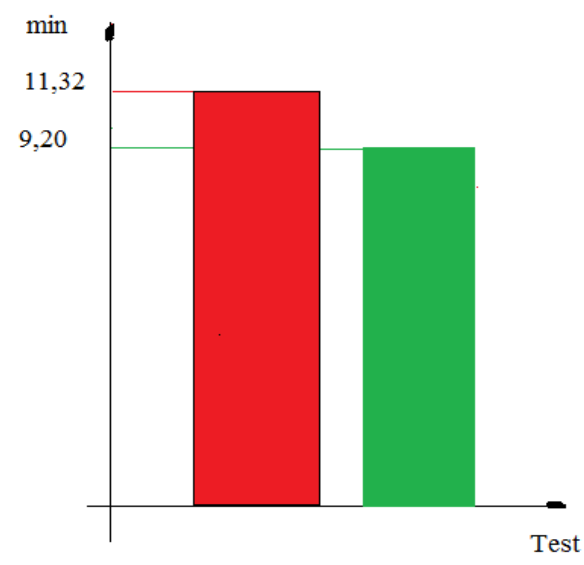

Fig. 13. The comparison time of manufacturing.

With NC BRAIN has high precision and best calculation speed in NC machining part.

Using NC BRAIN can help to do unmanned machining and reduce the machining time. And also NC BRAIN calculation speed is 6 times faster than other products.

The conclusions are, NC BRAIN give us a solution to optimize the NC program, the existed NC program. Is very important to do not make the mistake to conclude that the software will give us another technical solution to optimize the process. Will do not make change the milling strategies, will do not use another cutting tools, will be use what yet was establish.

NC BRAIN bring us what every good technical programmer know, to make a correct calculation of the feed, where you can add new passes, where you can take the passes what are not necessary, where are possible to grow up the positioning moving, where are not possible to do. This mean optimization - optimization what grow up our production time and make more safety our CNC manufacturing.

\section{References}

1. A. But, Machine and advance system of manufacturing (Ed. Politehnica Timisoara, 2009)

2. *** Metal Cutting Technology Technical guide

3. *** NC BRAIN MANUAL 